\title{
Spatial-Frequency Azimuthally Stable Cartography of Biological Polycrystalline Networks
}

\author{
V. A. Ushenko, N. D. Pavlyukovich, and L. Trifonyuk \\ Correlation Optics Department, Chernivtsi National University, 2 Kotsyubinsky Street, Chernivtsi 58012, Ukraine \\ Correspondence should be addressed to V. A. Ushenko; yuriyu@gmail.com
}

Received 1 May 2013; Revised 15 July 2013; Accepted 1 August 2013

Academic Editor: Nicusor Iftimia

Copyright ( 2013 V. A. Ushenko et al. This is an open access article distributed under the Creative Commons Attribution License, which permits unrestricted use, distribution, and reproduction in any medium, provided the original work is properly cited.

\begin{abstract}
A new azimuthally stable polarimetric technique processing microscopic images of optically anisotropic structures of biological tissues histological sections is proposed. It has been used as a generalized model of phase anisotropy definition of biological tissues by using superposition of Mueller matrices of linear birefringence and optical activity. The matrix element $M_{44}$ has been chosen as the main information parameter, whose value is independent of the rotation angle of both sample and probing beam polarization plane. For the first time, the technique of concerted spatial-frequency filtration has been used in order to separate the manifestation of linear birefringence and optical activity. Thereupon, the method of azimuthally stable spatial-frequency cartography of biological tissues histological sections has been elaborated. As the analyzing tool, complex statistic, correlation, and fractal analysis of coordinate distributions of $M_{44}$ element has been performed. The possibility of using the biopsy of the uterine wall tissue in order to differentiate benign (fibromyoma) and malignant (adenocarcinoma) conditions has been estimated.
\end{abstract}

\section{Introduction}

The state of the art in medical science demands from the experts not only certain practical skills but also knowledge of general pathologic processes, their nomenclature and definitions, causes, pathogenesis, and results as well as their importance for human. The pathologic processes on a cellular and tissue level can be detected by means of light microscope.

Samples preparation for microscopic investigations is a complex process that involves degradation of image quality of histological samples. In order to overcome the problems connected with image deformation (artifacts), the high professionalism and complex chore are necessary for results correction. The usage of computing for the mentioned tasks allows facilitating the work with medical images.

The methods and algorithms of objects isolation in the microscopic images-segmentation of histological objects in grayscale and color images-are mainly developing at present time. The algorithms of segmentation of extended (vessels, fibers) and areal (cells and their structures) objects are being elaborated [1-8].

A new medical-physical method, namely, laser polarimetry of biological tissues histological sections, becomes an extension and an addition to the techniques of microscopic images structure processing [9-11]. The background of such method consists in measurements of coordinate distributions of polarization states (polarization maps) in the plane of microscopic images of biological tissues histological sections. Such method allows obtaining a new, inaccessible measurement for histological and mathematical methods of analysis and information about optical anisotropy (linear and circular birefringence [11]) of nonuniformly scaled structural elements of different biological objects. At the same time, a complex analysis of the polarization maps of a tissue specimen is azimuthally dependent on the probing beam polarization plane and sample rotation angle. Such a condition complicates using such a method during the comparative investigations of groups of histological sections with different pathologies.

Thus, further progress of laser polarimetry may be connected with the development of azimuthally stable methods of direct measurement of the parameters of linear and circular birefringences.

The solution of this task is the use of Mueller-matrix cartography with the so-called rotational invariants. The Mueller 
matrix elements $M_{44}(\Theta)=$ const appear to be azimuthally stable and sample rotation angle $(\Theta)$ independent [12-15].

It has been determined that, in the case of optically thin (attenuation coefficient $\tau \leq 0.1$ ) layers, the $M_{44}(\Theta)$ value is preferably defined by phase anisotropy mechanisms-linear and circular birefringences [16].

The separation of such mechanisms $\left(M_{44} \Rightarrow\left(\begin{array}{c}M_{44} \text {-linear } \\ M_{44} \text {-circular }\end{array}\right)\right)$ is possible with the help of spatialfrequency Fourier selection [17].

Our research is aimed at designing the experimental method of azimuthally stable Fourier polarimetry and spatial-frequency selection of parameter distributions of linear and circular birefringences of the biopsy of the uterine wall tissue in order to differentiate benign (fibromyoma) and malignant (adenocarcinoma) conditions.

\section{The Theory of Method}

The following model concepts have been assumed [9-11] by us as the basis for an analysis of the processes of modulation of laser radiation by the polycrystalline network of biological crystals with linear and circular birefringences:

(i) the uterine wall tissue consists of two optical anisotropic layers of the muscular (myometrium) and connective (endometrium) layers;

(ii) the optical anisotropic myometrium network is formed by large-scale (the range of the transverse dimensions $l \sim 30 \mu \mathrm{m}-100 \mu \mathrm{m}$ ) filiform myosin fibrils with a prevalence by linear birefringence;

(iii) the optical anisotropy of such structures is characterized by coordinate distribution of the matrix element $M_{44}(\rho, \delta)$ in the plane of histological section, where $\rho$ is a direction of fibrils packing and $\delta$ is a phase shift between linearly polarized orthogonal components of the light beam amplitude;

(iv) the optical anisotropic component of the endometrium is formed by collagen fibers regulated according to the direction of optic axes $(l \sim 5 \mu \mathrm{m}-10 \mu \mathrm{m} ; L \approx l)$ with predominantly circular birefringence $M_{44}(\theta)$, where $\theta$ is polarization plane rotation angle.

From the medical point of view, "optical separation" of the polarization manifestations of linear and circular birefringences of optical anisotropic layers of the uterine wall is the topical task.

The fact is that, at the initial, early stages, the pathological changes in the human organism are accompanied by a concentration increase of optically active proteins in case of the generalized preservation of the morphological macrostructures of polycrystalline protein network of the myometrium and endometrium $[16,17]$.

Fibrillar structural changes - the formation of the growth direction of tumors shoots and so on-correspond to a more developed pathological condition.

With the purpose of a selection of such conditions, we have applied the method of spatial-frequency filtration of the polarization inhomogeneous field of laser radiation in Fourier plane $[18,19]$.

The main idea of such approach is that spatial-frequency structure of Fourier form of laser image of the tissue of the uterine wall is different for its large-scale (myosin) and smallscale (collagen) protein structures. Proceeding from this, using spatial-frequency filtration, it is mainly possible to isolate either low-frequency (with linear birefringence) or highfrequency (with circular birefringence) components, which by means of Fourier conversion transform into, respectively, "separated" laser images.

\section{Optical Realization of Spatial Frequency of Fourier Selection of the Uterine Tissue Optical Anisotropy of Protein Networks}

Figure 1 presents a diagram of modified laser Fourier polarimeter with spatial-frequency filtration [20].

Illumination of a sample under study was performed by the parallel $\left(\phi=10^{4}\right)$ laser beam of $\mathrm{He}-\mathrm{Ne}(\lambda=$ $0.6328 \mu \mathrm{m}, W=5.0 \mathrm{~mW})$. The polarization light source consisted of quarter-wave plates 3 and 5 and polarizer 4; it formed a right circularly polarized beam. The histological sections of the biopsy of the uterine wall tissue were placed in the focal plane of polarization microobjective 7 (focal distance-30 $\mathrm{mm}$, aperture-0.1, magnification-4x). Behind the Fourier focal plane, the vignetting diaphragm was located; its size changed within the range of 2 pix to 300 pix. Polarization microobjective 8 (focal distance$30 \mathrm{~mm}$, aperture -0.1 , and magnification $-4 \mathrm{x}$ ) was located at the focal length forming the frequency plane of lens 7 and, thus, performed inverse Fourier transform of a filtered image outside the polarization field of laser radiation. The coordinate distribution of intensity of such fields, polarizationally filtered by quarter-wave plate 9 and polarizer 10, was registered in the plane of CCD-camera 11 (the Imaging Source DMK 41AU02.AS, monochrome 1/2" CCD, Sony ICX205AL (progressive scan); resolution $-1280 \times 960$; light sensitive area size $-7600 \times 6200 \mu \mathrm{m}$; sensitivity $-0.05 \mathrm{~lx}$; dynamic range8 bit; SNR - 9 bit, deviation of photosensitive characteristics from linear no more than $15 \%$ ). It provided the range of measuring the structural elements of polycrystalline network with the resolution of $2-2000 \mu \mathrm{m}$.

The matrix element $M_{44}$ was calculated for each probing beam within every pixel $(m \times n)$ as follows:

$$
M_{44}=\frac{I_{\otimes}^{\otimes}-I_{\oplus}^{\otimes}}{I_{\otimes}^{\otimes}+I_{\oplus}^{\otimes}}-0.5\left(\frac{I_{\otimes}^{0}-I_{\oplus}^{0}}{I_{\otimes}^{0}+I_{\oplus}^{0}}+\frac{I_{\otimes}^{90}-I_{\oplus}^{90}}{I_{\otimes}^{90}+I_{\oplus}^{90}}\right) .
$$

Here, $I_{\otimes}^{\otimes ; 0 ; 90}$ and $I_{\oplus}^{\otimes ; 0 ; 90}$ are the intensities of spatialfrequency filtered image $(\otimes ; \oplus)$ for each polarization states of probing beams $\left(\otimes ; 0^{\circ} ; 90^{\circ}\right)$.

In order to get a quantitative estimation of coordinate distributions $q(m \times n) \equiv M_{44}$, we have used, as in the case of traditional laser polarimetry [9-11, 16, 17], three mutually complementary techniques of mathematical analysis, namely, statistic, autocorrelation, and fractal. 


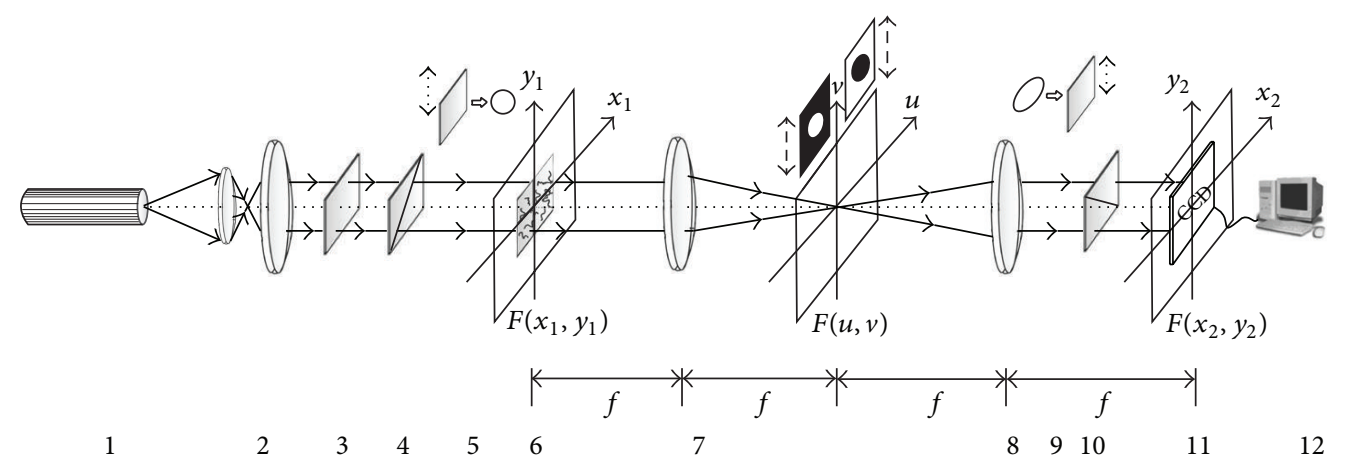

FIGURE 1: Optical scheme of Fourier polarimeter: 1-He-Ne laser, 2-a collimator, 3-a stationary quarter-wave plate, 5, 9-mechanically movable quarter-wave plates, 4, 10-a polarizer and analyzer, respectively, 6-object of study 7, 8-polarization microobjectives, 11-CCD camera, 12-a personal computer. $F\left(x_{1}, y_{1}\right)$-object plane, $F\left(x_{2}, y_{2}\right)$-image plane, and $F(u, v)$-Fourier plane with the diaphragms.

We have calculated a set of statistical moments of the 1st4th orders $Z_{j=1 ; 2 ; 3 ; 4}$ using the following algorithms:

$$
\begin{aligned}
& Z_{1}=\frac{1}{N} \sum_{i=1}^{N}\left|(q)_{i}\right|, \quad Z_{2}=\sqrt{\frac{1}{N} \sum_{i=1}^{N}(q)_{i}^{2}} \\
& Z_{3}=\frac{1}{\left(Z_{2}\right)^{3}} \frac{1}{N} \sum_{i=1}^{N}(q)_{i}^{3}, \quad Z_{4}=\frac{1}{\left(Z_{2}\right)^{4}} \frac{1}{N} \sum_{i=1}^{N}(q)_{i}^{4} .
\end{aligned}
$$

The obtained data characterizes the histogram of the element $M_{44}$ of optically anisotropic structures in the plane of histological section.

The method of autocorrelation with the use of function was assumed as the basis of an analysis of the coordinate structure of distributions $q(m \times n)$

$$
K_{i=1 \cdots n}(\Delta m)=\lim _{m \rightarrow 0} \frac{1}{m} \int_{1}^{m}\left[(q)_{i}(m)\right]\left[(q)_{i}(m-\Delta m)\right] d m .
$$

Here, $(\Delta m=1$ pix $)$ is the step of changing the coordinates $(x=1 \cdots m)$ of a distribution of a set of polarization distributions $q(m)$ for a specific $i$ th horizontal line of the pixel digital camera. The resultant expression for the autocorrelation function is obtained by averaging expression (3) along all lines $i=1 \cdots n$

$$
K(\Delta m)=\frac{\sum_{i=1}^{n} K_{i}(\Delta m)}{n} .
$$

The obtained dependency characterizes the coordinate interconnection between the optically anisotropic structures in the plane of histological section.

We have selected correlation moments $Q_{2}$ and $Q_{4}$ that determine a distribution and the excess of the autocorrelation function $K(\Delta m)$ as quantitative parameters that characterize autocorrelation dependences $K(\Delta m)$.

A fractal analysis of $q(m \times n)$ distributions was performed using a calculation of logarithmic dependences $\log J(q)-\log v$ for the power spectra $J(q)$ with following evaluation of $D$ dispersion.
Dependences $\log J(q)-\log v$ are approximated using the least-squares method into curves $V(\eta)$ and are classified in such a way:

(i) $q(m \times n)$ are fractal on condition that the value of the slope is constant $\eta=$ constant for 2-3 decades of a change of sizes $l$;

(ii) $q(m \times n)$ are multifractal, on condition of the presence of several slopes in $V(\eta)$;

(iii) $q(m \times n)$ are random, in case of the absence of stable slopes $V(\eta)$ over the whole range of a change sizes $l$.

The obtained results characterize the scale self-similarity of the distribution of optically anisotropic structures in the plane of histological section.

\section{Experimental Results and Discussion}

As objects of investigation, we chose two groups of opticallythin (attenuation coefficient $\tau \approx 0.087-0.098$ ), single scattered histological sections of the biopsy of benign (16 samples) and malignant (14 samples) tumors of the uterus.

The samples were prepared on a freezing microtome, using a standard technique. From the optical point of view, such samples characterized by transformation of polarization without depolarization.

The conventional microscopic images of the samples of both groups are presented in Figure 2.

As it can be seen from Figure 2, the coordinate and scale structures of such conventional microscopic images are similar. This fact considerably complicates the histological differentiation of benign and malignant states of uterus tissue.

4.1. Spatial-Frequency Fourier Polarimetry of Linear Birefringence of the Fibrillar Matrices of the Uterine Tissue. For the purpose of choosing the optimal conditions of spatialfrequency filtration, the following range $\Delta r=2$ pix-50 pix of possible sizes of the vignetting diaphragm was used.

The criterion for diaphragm size choosing is a simultaneous change of the set of statistic moments $Z_{j=1 ; 2 ; 3 ; 4}(4)$ of $M_{44}$. In our case, the optimal size was $\Delta r=30$ pix. 


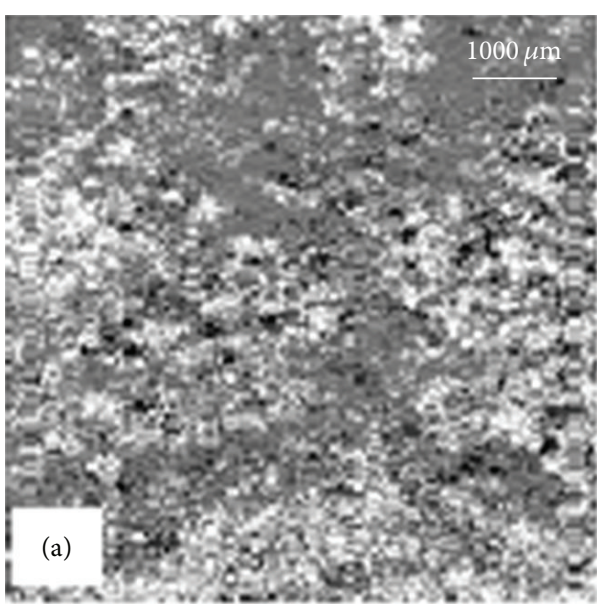

(a)

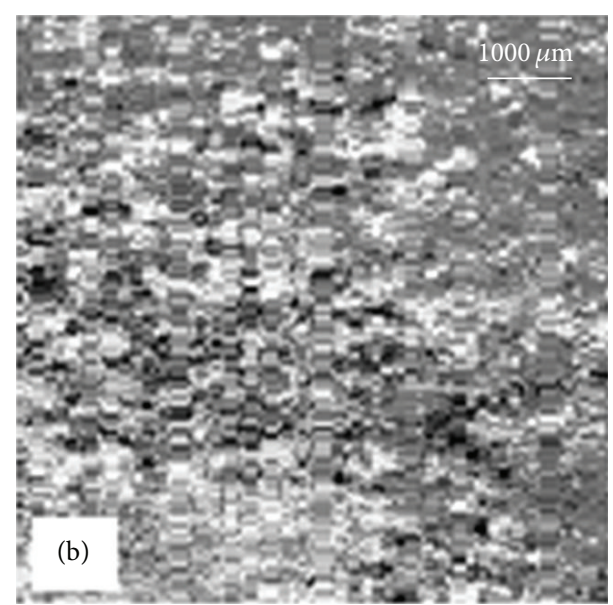

(b)

FIGURE 2: Microscopic images of histological sections of the biopsy of benign (a) and malignant (b) tumors of the uterus.

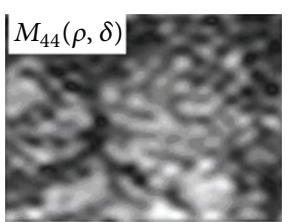

(a)

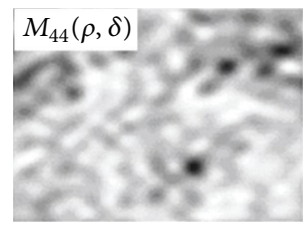

(e)

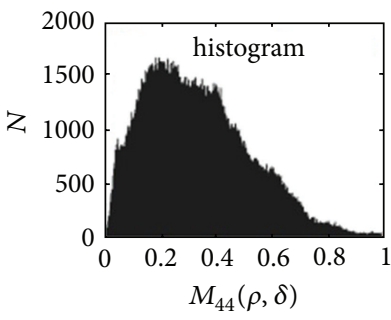

(b)

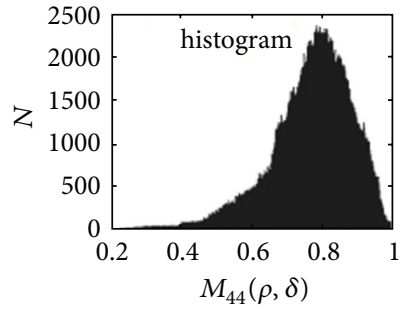

(f)

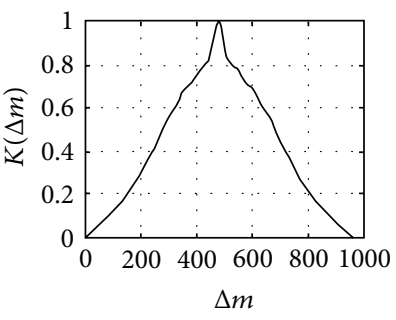

(c)

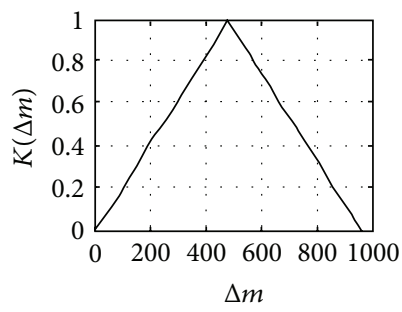

(g)

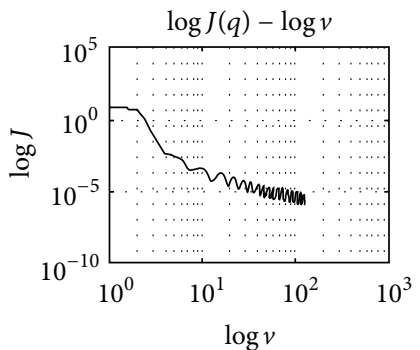

(d)

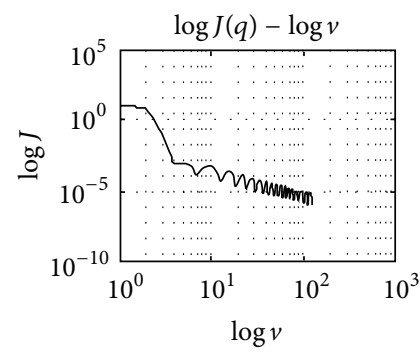

(h)

FIGURE 3: "Low-frequency" coordinate maps $M_{44}(\rho, \delta)$ (a), (e) histograms (b), (f) autocorrelation functions (c), (g) logarithmic dependence of the power spectra $(\mathrm{d}),(\mathrm{h})$ of the linear birefringence distribution of a histological section of benign uterine tumor (fibromyoma) biopsy $((\mathrm{a})-(\mathrm{d}))$, and malignant uterine tumor (adenocarcinoma) biopsy $((\mathrm{e})-(\mathrm{h})$ ).

Such geometric size was chosen for comparative investigations of optical anisotropy of the fibrillar networks of the uterine tissue with benign and malignant changes, which characterize coordinate distributions of rotational invariant $M_{44}(\rho, \delta)$-Figure 3 .

A comparative analysis of aggregate parameters that characterize matrix maps $M_{44}(\rho, \delta)$ of linear birefringence of large-scale optically anisotropic fibrillar networks of the uterine tissue samples revealed some discrepancies between them. Namely, the main extrema of histograms of the distributions of random matrix maps, $M_{44}(\rho, \delta)$ values for histological sections of both types are localized in different areas. Thus, the most probable value is $M_{44}(\rho, \delta) \sim 0.2$ for a benign tumor (Figure 3(b)), for a malignant one it is $M_{44}(\rho, \delta) \sim 0.8$ (Figure 3(f)). The revealed peculiarity, in our opinion, is connected with a more developed structure of newly formed fibers of malignant tumor than with benign fibromyoma of the similar optical anisotropic network.

Dependences of autocorrelation functions $K(\Delta m)$ (4) of $M_{44}(\rho, \delta)$ distribution of both uterine tissue samples smoothly and monotonously, (Figure 3, fragments (c) and (g)) indicate a coordinately homogeneous structure of the corresponding matrix maps formed by the mechanisms of linear birefringence of myosin fibrils. Besid such maps, 
TABLE 1: Parameters of the statistic, correlation, and self-similar structure of $M_{44}(\rho, \delta)$ of linear birefringence of polycrystalline uterine tissue networks.

\begin{tabular}{lccc}
\hline \multirow{2}{*}{ Parameters } & \multicolumn{2}{c}{$M_{44}(\rho, \delta)$} & Sp \\
& Fibromyoma & Adenocarcinoma & \\
\hline$Z_{1}$ & $\mathbf{0 . 3 3} \pm \mathbf{0 . 0 5 9}$ & $\mathbf{0 . 7 2} \pm \mathbf{0 . 1 1}$ & $72 \%$ \\
$Z_{2}$ & $\mathbf{0 . 1 4} \pm \mathbf{0 . 0 2 2}$ & $\mathbf{0 . 1 8} \pm \mathbf{0 . 0 3 1}$ & $61 \%$ \\
$Z_{3}$ & $\mathbf{2 . 1 6} \pm \mathbf{0 . 3 7}$ & $\mathbf{0 . 8 3} \pm \mathbf{0 . 1 4}$ & $75 \%$ \\
$Z_{4}$ & $\mathbf{1 . 9 5} \pm \mathbf{0 . 2 1}$ & $\mathbf{0 . 3 7} \pm \mathbf{0 . 0 5 1}$ & $77 \%$ \\
$Q_{2}$ & $0.31 \pm 0.049$ & $0.33 \pm 0.054$ & $56 \%$ \\
$Q_{4}$ & $0.035 \pm 0.005$ & $0.04 \pm 0.006$ & $54 \%$ \\
$V(\eta)$ & Fractal & Fractal & $51 \%$ \\
$D$ & $0.33 \pm 0.055$ & $0.28 \pm 0.051$ & $52 \%$ \\
\hline
\end{tabular}

a dimensionally self-similar construction has been deletedlogarithmic dependences of power distributions spectra $M_{44}(\rho, \delta)$ are characterized by a similar slope (Figure 3, fragments $(\mathrm{d}),(\mathrm{h}))$.

Thus, matrix maps $M_{44}(\rho, \delta)$ (Figure 3, fragments (a) and (e)) of linear birefringence of the polycrystalline network of myosin fibrils are characterized by a sufficiently similar correlation and homogeneous (fractal) structure. The main indication of oncologic changes is the formation of a developed optically anisotropic network with an increased level of linear birefringence index and a transformation of a histogram of the distribution of $M_{44}(\rho, \delta)$.

Quantitative differences between matrix maps $M_{44}(\rho, \delta)$ of the protein matrices of the uterine tissue of both types illustrate average values and standard deviations of the set of statistic $Z_{i=1 ; 2 ; 3 ; 4}$, correlative $\left(Q_{i=2 ; 4}\right)$, and fractal $(D)$ parameters, illustrated in Table 1 . Also, let us introduce the specificity parameter $\mathrm{Sp}=a(a+b)^{-1}(a, b$-the number of the correct and incorrect diagnoses within a group) in differentiation of benign and malignant uterine tumors.

A comparative analysis of the data of laser-frequency Fourier-polarimetry of linear birefringence of protein polycrystalline networks of histological section of benign and malignant uterine tumors biopsy revealed sensibility to a differentiation of such states of all statistic moments of the 1st through the 4 th orders with specificity level Sp 60\%-77\% (printed in bold in Table 1).

4.2. Spatial-Frequency Fourier Polarimetry of Circular Birefringence of the Fibrillar Networks of Uterine Tissue. Diagnostic possibilities of differentiating of pathologically changed samples of the uterine tissue, using the method of highfrequency spatial-frequency Fourier-domain-polarimetry of circular birefringence employing opaque filter illustrates probable (fragments (b), (f)), autocorrelation (fragments (c), (g)), and spectral (fragments (d), (h)) dependences of matrix maps $M_{44}(\theta)$ (fragments (a), (e)), adduced in a series in Figure 4.

On comparing the findings about the structure of the distributions of circular birefringence index (Figure 4, fragments (a) and (e)) under the conditions of high frequency
TABLE 2: Parameters of the statistic, correlation, and self-similar structure of $M_{44}(\theta)$ of circular birefringence of polycrystalline uterine tissue networks.

\begin{tabular}{lccc}
\hline \multirow{2}{*}{ Parameters } & \multicolumn{2}{c}{$M_{44}(\theta)$} & Sp \\
& Fibromyoma & Adenocarcinoma & \\
\hline$Z_{1}$ & $\mathbf{0 . 1 3} \pm \mathbf{0 . 0 2 1}$ & $\mathbf{0 . 2 4} \pm \mathbf{0 . 0 3 7}$ & $\mathbf{7 5 \%}$ \\
$Z_{2}$ & $\mathbf{0 . 1 1} \pm \mathbf{0 . 0 1 8}$ & $\mathbf{0 . 2 2} \pm \mathbf{0 . 0 3 5}$ & $\mathbf{8 1 \%}$ \\
$Z_{3}$ & $\mathbf{0 . 7 4} \pm \mathbf{0 . 1 1}$ & $\mathbf{2 . 3 8} \pm \mathbf{0 . 3 9}$ & $\mathbf{8 7 \%}$ \\
$Z_{4}$ & $\mathbf{0 . 8 9} \pm \mathbf{0 . 1 3}$ & $\mathbf{1 . 5 8} \pm \mathbf{0 . 2 1}$ & $\mathbf{8 4 \%}$ \\
$Q_{2}$ & $0.19 \pm 0.031$ & $0.23 \pm 0.034$ & $53 \%$ \\
$Q_{4}$ & $\mathbf{1 . 5 6} \pm \mathbf{0 . 2 8}$ & $\mathbf{0 . 7 6} \pm \mathbf{0 . 1 2}$ & $\mathbf{7 2 \%}$ \\
$V(\eta)$ & Multifractal & Fractal & $\mathbf{7 3 \%}$ \\
$D$ & $0.31 \pm 0.059$ & $0.28 \pm 0.047$ & $51 \%$ \\
\hline
\end{tabular}

filtration of laser field radiation, transformed by polycrystalline collagen fibrils of a layer of the endometrial tissue are the following characteristics of the oncologic state, detected. Firstly, it is a range expansion of random values that changes $M_{44}(\theta)$ for a histogram of the matrix map at the expense of a "pathologic" increase of the concentrations and the size of the collagenous fibrils (Figure 4, fragments (b), (f)). Secondly, the formation of growth orientations leads to the transformation of the multifractal (Figure 4, fragment (d)) distribution $M_{44}(\theta)$ into a self-similar-inherent fixed inclination $\eta$ (Figure 4, fragment (h) for approximate curve $V(\eta))$.

Quantitative differences between matrix maps $M_{44}(\theta)$ of circular birefringence of polycrystalline collagenous networks of the endometrium with pathology of both types illustrate average values and standard deviations of statistic set $Z_{i=1 ; 2 ; 3 ; 4}$, correlative $\left(Q_{i=2 ; 4}\right)$, and fractal $(D)$ parameters, presented in Table 2 .

A comparative analysis revealed the following oncologic condition, with a high level of specificity $(72 \% \leq \mathrm{Sp} \leq 87 \%)$ of uterus parameters (printed in bold in Table 2):

(i) statistic moments of the 1st and the 2 nd orders $\theta(m \times$ $n)$; differences between values are from 2 to 3,5 times;

(ii) correlation moments of the 4 th order of the autocorrelation function of distributions $M_{44}(\theta)$; differences between the values reach 2 times;

(iii) transformation of multifractal distributions $M_{44}(\theta)$ into fractal ones.

\section{Conclusions}

A model of generalized optic anisotropy of the fibrillar protein matrices has been suggested, and the method of Fourier polarimetry for the parameters of linear (phase shift between orthogonal components of the laser wave amplitude) and circular (the rotation angle of the polarization plane) birefringences of the tissue biopsy of the uterine wall a spatial frequency selection of such coordinate distributions for a differentiation of benign (fibromyoma) and malignant (adenocarcinoma) conditions has been substantiated. 


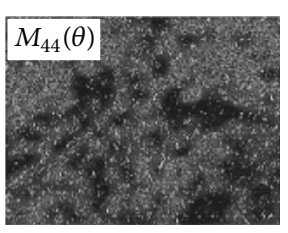

(a)

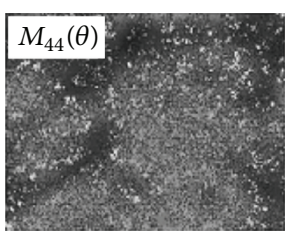

(e)

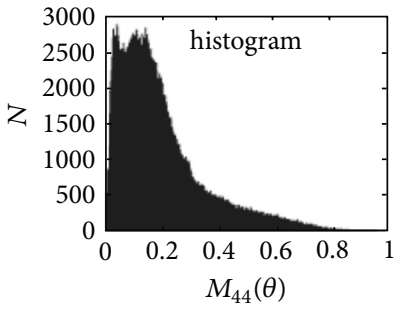

(b)

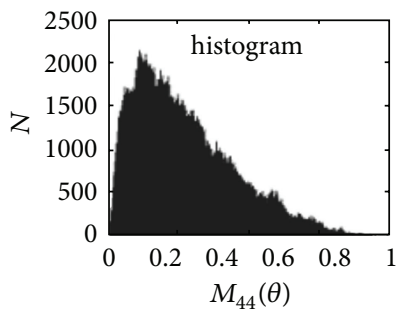

(f)

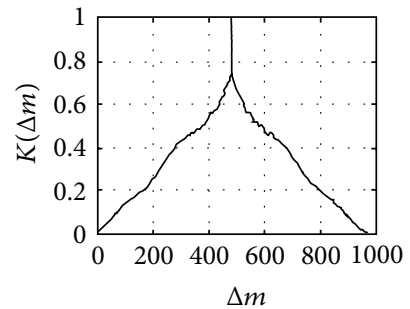

(c)

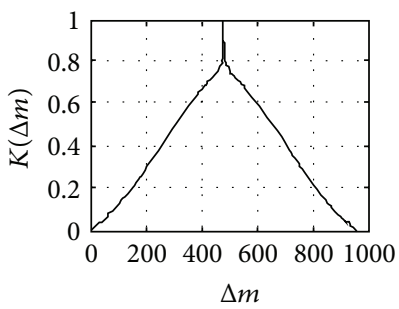

(g)

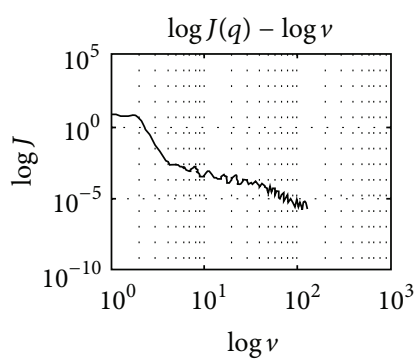

(d)

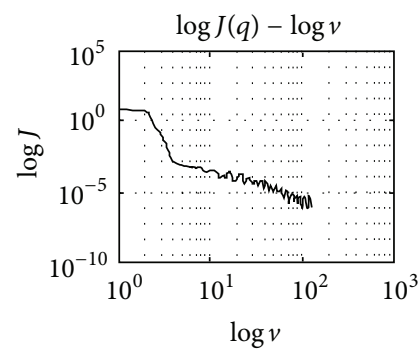

(h)

Figure 4: "High-frequency" coordinate maps $M_{44}(\theta)$ (a), (e) histograms (b), (f) autocorrelation functions (c), (g) logarithmic dependence of the power spectra $(\mathrm{d}),(\mathrm{h})$ of the linear birefringence distribution of a histological section of benign uterine tumor (fibromyoma) biopsy ((a)-(d)), and malignant uterine tumor (adenocarcinoma) biopsy ((e)-(h)).

A set of criteria for a polarization-phase differentiation of benign (fibromyoma) and malignant (adenocarcinoma) conditions of the uterine has been revealed and substantiated as follows:

(i) statistical moments of the 1st-4th order which characterize a distribution of azimuthally stable matrix element $M_{44}(\rho, \delta)$, stipulated by linear birefringence of myosin fibrils of the endometrium layer;

(ii) statistical moments of the 1st-4th orders which characterize a distribution of azimuthally stable matrix element $M_{44}(\theta)$, stipulated by circular birefringence of the network of collagenous fibrils of the endometrium layer;

(iii) correlation moments of the 4 th order, which characterize autocorrelation functions of the distributions for $M_{44}(\theta)$, stipulated by circular birefringence of the network of collagenous fibrils;

(iv) a slope change of approximate curve of log-log dependency for the power spectra of distribution of $M_{44}(\rho, \delta)$ and $M_{44}(\theta)$ transformed by optical anisotropic protein matrices.

\section{References}

[1] K. Abe, F. Mizutani, and C. Wang, "Thinning of gray-scale images with combined sequential and parallel conditions for pixel removal," IEEE Transactions on Systems, Man and Cybernetics, vol. 24, no. 2, pp. 294-299, 1994.

[2] C. Arcelli and G. Ramella, "Sketching a grey-tone pattern from its distance transform," Pattern Recognition, vol. 29, no. 12, pp. 2033-2045, 1996.
[3] C. Arcelli and G. Ramella, "Finding grey-skeletons by iterated pixel removal," Image and Vision Computing, vol. 13, no. 3, pp. 159-167, 1995.

[4] A. Bieniek and A. Moga, "A connected component approach to the watershed segmentation," in Mathematical Morphology and Applications to Image and Signal Processing, H. J. A. M. Heijmans and J. B. T. M. Roerdink, Eds., pp. 215-222, Kluwer Academic publishers, London, UK, 1998.

[5] R. Boomgaard, Mathematical morphology: extension towards computer vision [Academisch proefschrift], Faculteit der Wiskunde en Informatica de Universiteit van Amsterdam, 1992.

[6] L. E. Bracey Jr., "Image analysis for nanotechnology on the desktop," Advanced Imaging, vol. 12, no. 11, pp. 54-55, 1997.

[7] A. Nedzved and S. Ablameyko, "Extraction on cell structure in color histological images," in Proceedings of the International Workshop on Computer Vision, Computer Graphics, New Media, pp. 41-49, Computer Gesellschaft, Graz, Austria, 2002.

[8] A. M. Nedzved, S. V. Ablameyko, B. V. Nalibotsky, and Y. G. Iliych, "System for analysis of medical images of histological objects," Pattern Recognition and Image Analysis, vol. 11, no. 4, pp. 732-742, 2001.

[9] O. V. Angelsky, A. G. Ushenko, A. Yu. Ushenko, V. P. Pishak, and A. P. Peresunko, "Statistical, correlation and topological approaches in diagnostics of the structure and physiological state of birefringent biological tissues," in Handbook of Photonics for Biomedical Science, V. V. Tuchin, Ed., pp. 283-322, CRC Press, Taylor \& Francis Group, Boca Raton, Fla, USA, 2010.

[10] O. V. Angelsky, V. P. Pishak, A. G. Ushenko, and A. Ushenko, "Statistical and fractal structure of biological tissue Mueller matrix images," in Optical Correlation Techniques and Applications, O. Angelsky, Ed., vol. 6616 of Proceedings of SPIE, pp. 213263, 2007. 
[11] Y. A. Ushenko, T. M. Boychuk, V. T. Bachynsky, and O. P. Mincer, "Diagnostics of structure and physiological state of birefringent biological tissues: statistical, correlation and topological approaches," in Handbook of Coherent-Domain Optical Methods, pp. 107-148, Springer Science, New York, NY, USA, 2013.

[12] J. M. Bueno and J. Jaronski, "Spatially resolved polarization properties for in vitro corneas," Ophthalmic and Physiological Optics, vol. 21, no. 5, pp. 384-392, 2001.

[13] M. Shribak and R. Oldenbourg, "Techniques for fast and sensitive measurements of two-dimensional birefringence distributions," Applied Optics, vol. 42, no. 16, pp. 3009-3017, 2003.

[14] M. H. Smith, "Interpreting Mueller matrix images of tissues," in Laser-Tissue Interaction XII: Photochemical, Photothermal, and Photomechanical, vol. 4257 of Proceedings of SPIE, pp. 82-89, San Jose, Calif, USA, January 2001.

[15] S.-Y. Lu and R. A. Chipman, "Interpretation of Mueller matrices based on polar decomposition," Journal of the Optical Society of America A, vol. 13, no. 5, pp. 1106-1113, 1996.

[16] O. V. Angelsky, Y. Y. Tomka, A. G. Ushenko, Y. G. Ushenko, and Y. A. Ushenko, "Investigation of 2D Mueller matrix structure of biological tissues for pre-clinical diagnostics of their pathological states," Journal of Physics D, vol. 38, no. 23, pp. 4227-4235, 2005.

[17] A. G. Ushenko, D. N. Burkovets, and Y. A. Ushenko, "Polarization-phase mapping and reconstruction of biological tissue architectonics during diagnosis of pathological lesions," Optics and Spectroscopy, vol. 93, no. 3, pp. 449-456, 2002.

[18] J. W. Goodman, "Statistical properties of laser speckle patters," in Laser Speckle and Related Phenomena, J. C. Dainty, Ed., pp. 9-75, Springer, Berlin, Germany, 1975.

[19] M. Born and E. Wolf, Principles of Optics, Cambridge University Press, Cambridge, UK, 1999.

[20] R. Józwicki, K. Patorski, O. V. Angelsky, A. G. Ushenko, D. N. Burkovets, and Y. A. Ushenko, "Automatic polarimetric system for early medical diagnosis by biotissue testing," Optica Applicata, vol. 32, no. 4, pp. 603-612, 2003. 

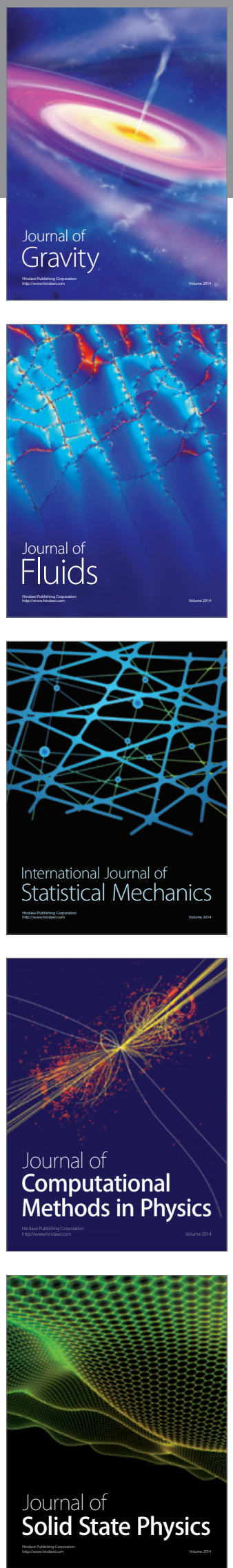

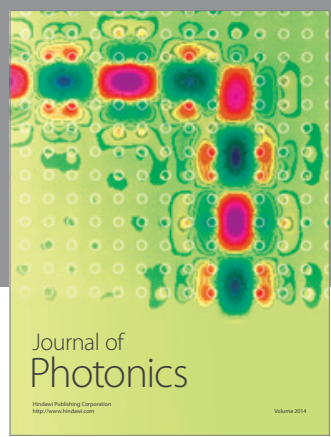

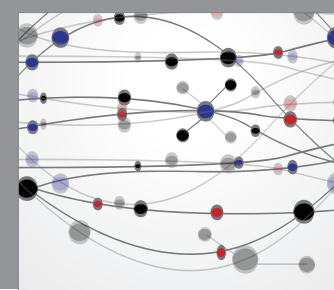

The Scientific World Journal

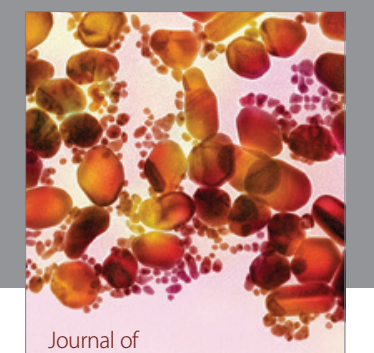

Soft Matter
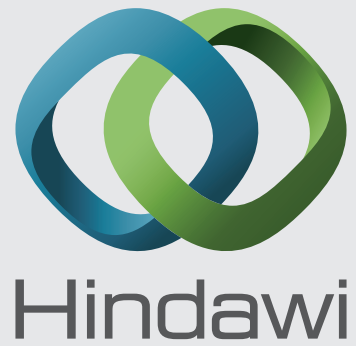

Submit your manuscripts at

http://www.hindawi.com
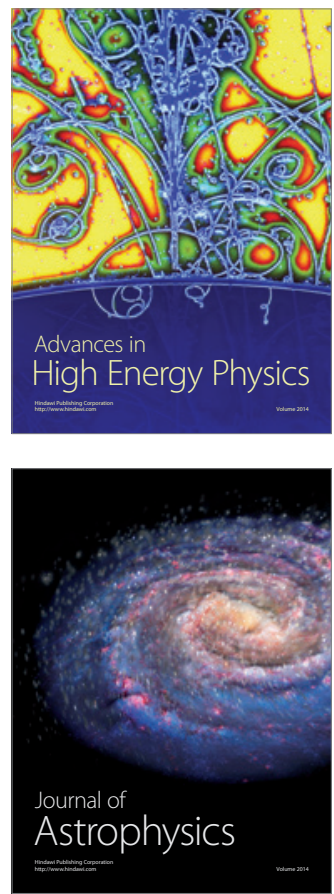
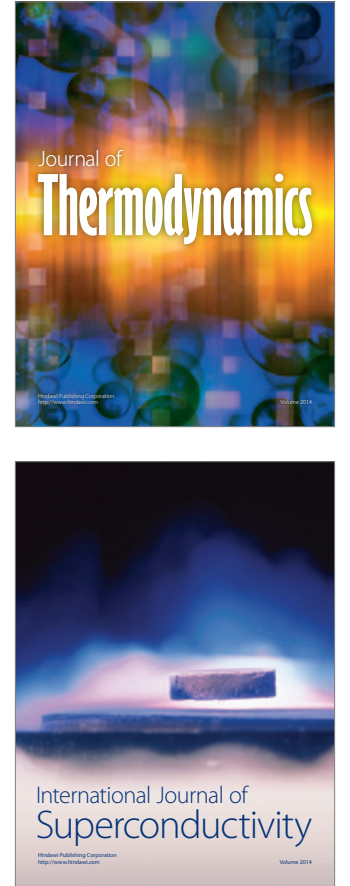
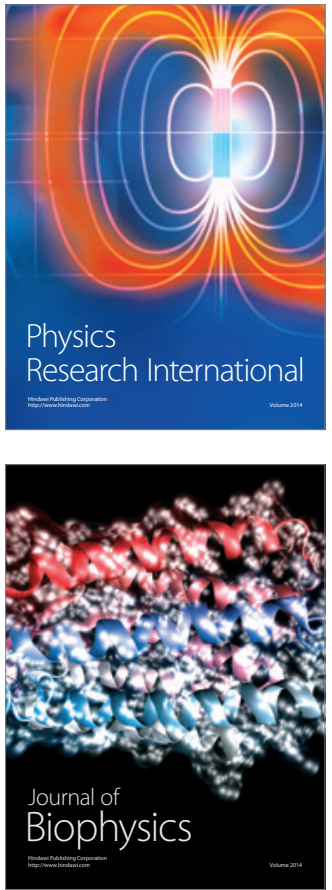
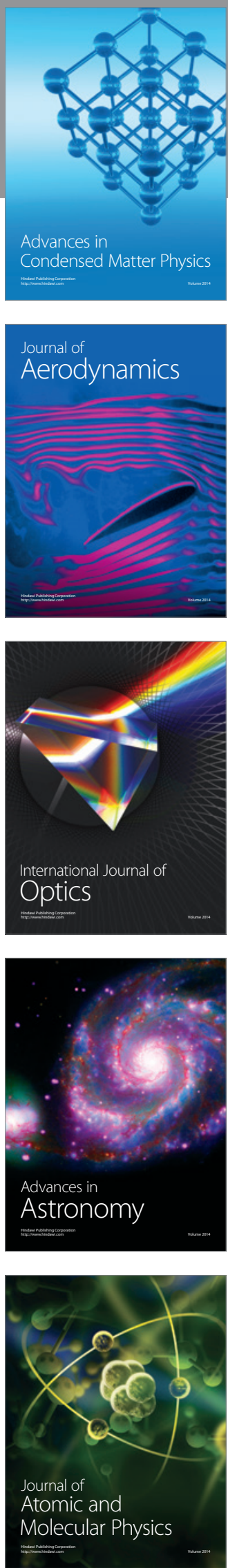